\title{
A Relativistic Chiral Quark Model For Pseudoscalar Emission From Heavy Mesons
}

\author{
J. L. Goity \\ Department of Physics, Hampton University, Hampton, VA 23668, USA \\ and \\ Thomas Jefferson National Accelerator Facility \\ 12000 Jefferson Avenue, Newport News, VA 23606, USA. \\ W. Roberts \\ Department of Physics, Old Dominion University, Norfolk, VA 23529, USA \\ and \\ Thomas Jefferson National Accelerator Facility \\ 12000 Jefferson Avenue, Newport News, VA 23606, USA.
}

\begin{abstract}
The amplitudes for one-pion mediated transitions between heavy meson excited states are obtained in the framework of the relativistic chiral quark model. The effective coupling constants to pions and the decay widths of excited heavy mesons with $\ell \leq 2$ for non-radially excited, and the $\ell=0$ radially excited mesons are presented for both charmed and beauty mesons. We also discuss the allowed decays of strange excited heavy mesons by emission of a K-meson.
\end{abstract}

JLAB-THY-98-36 


\section{INTRODUCTION}

It is known that the excited states of heavy mesons are of relevance in understanding the various weak decays of $B$ and $D$ mesons. One example is the process $B \rightarrow D e \nu$ where the excited heavy meson states influence the slope of the Isgur-Wise form factor [1] according to the Bjorken sum rule [2]. $B_{\ell 4}$ decays are another example where virtual excited states give rise to very important contributions to the total rates as well as to the shape of the partial width in the $D \pi$ invariant mass [3]. In most processes of interest these excited states couple to the ground state heavy meson by emission of one or more pions. This is the main reason why a good understanding of these couplings is necessary and important.

The properties of excited heavy mesons are also interesting in their own right, as new insight into strong interaction dynamics could be gained by studying such states. Again, in this process the knowledge of the pion couplings to the excited mesons is crucial. Thus far, only the $D_{1}$ and $D_{2}$ states have been determined experimentally [4]. These states decay by emitting a pion in a D-wave, and for this reason they are relatively narrow and could be observed. Other excited states are likely to be substantially wider and thus harder to be observed. It is important to provide estimates of these widths in order to judge the feasibility of such observations. This is one of our objectives here.

In this work we study the couplings of pions to heavy mesons and their excitations in the framework of the relativistic version of the chiral quark model of Georgi and Manohar [5]. We also obtain the decay rates of various lower excitations of heavy mesons. Comparison with previous results obtained in non-relativistic models is made and, in particular, the important relativistic effects noticed in the S-wave decay widths are emphasized. The possible decays of excited heavy mesons with strangeness via K meson emission are also discussed, and the interesting situation of some states that cannot decay in that way due to phase space is addressed.

Due to the heavy quark spin symmetry in the $M_{Q} \rightarrow \infty$ limit, heavy meson states come in nearly degenerate pairs of definite parity and with spins differing by one unit. In such a pair the light degrees of freedom (light quark plus glue, or in short, the brown muck) are in a state of angular momentum $j$ and parity $P$. Thus, all pairs have definite $j^{P}$ quantum numbers. In theory, the implementation of the spin symmetry is conveniently and elegantly made by associating superfields [6] with each pair. These superfields are expressed in terms of Dirac matrices, and the tensor fields (polarization tensors) required to describe the states in the doublet. There are two types of superfields depending on whether the parity is $P=(-1)^{j-\frac{1}{2}}$ or $P=(-1)^{j+\frac{1}{2}}$. Defining $k=j-\frac{1}{2}$, the superfields are

$$
\begin{aligned}
P=(-1)^{k} & : \\
\mathcal{H}_{-}^{\mu_{1} \ldots \mu_{k}} & =\frac{1+\not}{2 \sqrt{2}}\left\{Q_{j+1 / 2}^{\mu_{1} \ldots \mu_{k+1}} \gamma_{\mu_{k+1}}-\sqrt{\frac{2 k+1}{k+1}} \gamma_{5} Q_{j-1 / 2}^{\nu_{1} \ldots \nu_{k}}\left[g_{\nu_{1}}^{\mu_{1} \ldots g_{\nu_{k}}^{\mu_{k}}}\right.\right. \\
& \left.\left.-\frac{1}{2 k+1}\left(\gamma_{\nu_{1}}\left(\gamma^{\mu_{1}}-v^{\mu_{1}}\right) g_{\nu_{2}}^{\mu_{2}} \ldots g_{\nu_{k}}^{\mu_{k}}+\ldots+g_{\nu_{1} \ldots}^{\mu_{1}} \ldots g_{\nu_{k-1}}^{\mu_{k-1}} \gamma_{\nu_{k}}\left(\gamma^{\mu_{k}}-v^{\mu_{k}}\right)\right)\right]\right\} \\
P=(-1)^{k+1} & : \\
\mathcal{H}_{+}^{\mu_{1} \ldots \mu_{k}} & =\frac{1+\not p}{2 \sqrt{2}}\left\{Q_{j+1 / 2}^{\mu_{1} \ldots \mu_{k+1}} \gamma_{5} \gamma_{\mu_{k+1}}-\sqrt{\frac{2 k+1}{k+1}} Q_{j-1 / 2}^{\nu_{1} \ldots \nu_{k}}\left[g_{\nu_{1}}^{\mu_{1}} \ldots g_{\nu_{k}}^{\mu_{k}}\right.\right.
\end{aligned}
$$




$$
\left.\left.-\frac{1}{2 k+1}\left(\gamma_{\nu_{1}}\left(\gamma^{\mu_{1}}+v^{\mu_{1}}\right) g_{\nu_{2}}^{\mu_{2}} \ldots g_{\nu_{k}}^{\mu_{k}}+\ldots+g_{\nu_{1}}^{\mu_{1}} \ldots g_{\nu_{k-1}}^{\mu_{k-1}} \gamma_{\nu_{k}}\left(\gamma^{\mu_{k}}+v^{\mu_{k}}\right)\right)\right]\right\}
$$

These superfields are traceless, totally symmetric tensors that are transverse with respect to the four-velocity $v_{\mu}$. They also satisfy the constraint of being transverse to the Dirac $\gamma$ matrices. The spin-symmetry transformation law of the superfields is

$$
\begin{aligned}
\mathcal{H}^{\mu_{1} \ldots \mu_{k}} & \rightarrow \exp \left(-i \vec{\epsilon} \cdot \overrightarrow{S_{v}}\right) \mathcal{H}^{\mu_{1} \ldots \mu_{k}}, \\
S_{v}^{j} & =i \epsilon^{j k l}\left[\phi_{k}, \phi_{l}\right] \frac{(1+\not \varnothing)}{2},
\end{aligned}
$$

where the $e_{k}^{\mu}$ are space like vectors orthogonal to the four velocity. The conjugated superfields, which transform contravariantly under spin-symmetry, are simply given by $\overline{\mathcal{H}}^{\mu_{1} \ldots \mu_{k}}=\gamma_{0} \mathcal{H}^{\dagger \mu_{1} \ldots \mu_{k}} \gamma_{0}$. We are using here a normalization of the fields $Q^{\dagger}$ that is different from we used in our previous work [3]. The current normalization corresponds to the standard non-relativistic normalization.

The transition amplitudes of interest to us, namely the decay amplitudes of an excited heavy hadron into a heavy hadron and a light hadron, is proportional to

$$
(-1)^{\left(1 / 2+j^{\prime}+J_{h}+J\right)} \sqrt{\left(2 J^{\prime}+1\right)(2 j+1)}\left\{\begin{array}{ccc}
1 / 2 & j^{\prime} & J^{\prime} \\
J_{h} & J & j
\end{array}\right\}
$$

which arises from spin counting arguments [8]. The primed quantities above refer to the heavy daughter hadron, the unprimed ones refer to the parent, $J=j \pm 1 / 2, J^{\prime}=j^{\prime} \pm 1 / 2,\{\}$ is a $6-J$ symbol and $J_{h}=S_{h}+\ell_{h}$. $S_{h}$ is the total angular momentum of the light hadron $h$ and $\ell_{h}$ is the relative orbital angular momentum between $h$ and the heavy daughter produced in the decay. For the pion, $S_{h}=0$, so $J_{h}=\ell_{\pi}$.

Consistent with this, one approach that has been used for the description of the emission of a soft pion is HQET and ChPT combined into an effective theory [7]. In this combined effective theory, the couplings of pions to ground state mesons, for instance, is contained in the interaction term

$$
\mathcal{L}_{\chi}^{\text {int }}=g \operatorname{Tr}_{\mathrm{D}}\left(\overline{\mathcal{H}}_{-} \omega^{\mu} \mathcal{H}_{-} \gamma_{\mu} \gamma_{5}\right)
$$

where

$$
\omega_{\mu}=\frac{i}{2}\left(u \partial_{\mu} u^{\dagger}-u^{\dagger} \partial_{\mu} u\right)=\frac{1}{2 F_{\pi}} \partial_{\mu} \Pi+\ldots
$$

In the above, $\mathcal{H}$ is an $\mathrm{SU}(3)$ triplet, $\Pi=\pi^{a} \lambda^{a}$, the Gell-Mann matrices are normalized to $\operatorname{Tr}\left(\lambda^{a} \lambda^{b}\right)=2 \delta^{a b}, u=\exp \left(i \Pi / 2 F_{\pi}\right), F_{\pi}=93 \mathrm{MeV}$ is the pion decay constant, and $\operatorname{Tr}_{\mathrm{D}}$ denotes the trace over Dirac indices. Similarly, it is straightforward to write down terms that involve transitions among other spin-flavor multiplets.

In a more recent approach [9], the constraints of ChPT are dispensed with in favor of allowing for the emission of pions which are not necessarily soft. In the case of single pion emissions, for instance, this approach leads to an effective Lagrangian of the form

$$
\frac{1}{2 F_{\pi}} \operatorname{Tr}_{\mathrm{D}}\left[\overline{\mathcal{H}}^{\mu_{1} \ldots \mu_{k^{\prime}}} \lambda^{a} \mathcal{H}^{\nu_{1} \ldots \nu_{k}} \gamma_{5} A_{\mu_{1} \ldots \mu_{k^{\prime}}, \nu_{1} \ldots \nu_{k}} \pi^{a}\right]
$$


which is valid in the limit of $\mathrm{SU}(3)$. The factor of $1 /\left(2 F_{\pi}\right)$ is included here for dimensional reasons only. $A$ is a tensor of natural parity which is the most general one that can be built from Dirac matrices, the four velocity $v$ and the momentum of the pion. Implicit in this interaction Lagrangian are the parity and angular momentum selection rules in the heavy quark limit, $J^{P} \rightarrow J^{\prime P^{\prime}}+\ell_{\pi}, \ell_{\pi}+1=\left|P-P^{\prime}\right|_{\bmod 2},\left|j-j^{\prime}\right| \leq \ell_{\pi} \leq j+j^{\prime}$. For the transitions of interest to us we have

$$
\begin{aligned}
& \left(0^{-}, 1^{-}\right) \rightarrow\left(0^{-}, 1^{-}\right): \quad \mathrm{P} \text { - wave transition, } \\
& A=\alpha_{\left(0^{-}, 1^{-}\right) \rightarrow\left(0^{-}, 1^{-}\right)} \gamma_{\mu} \partial_{\pi}^{\mu}, \\
& g=-\alpha_{\left(0^{-}, 1^{-}\right) \rightarrow\left(0^{-}, 1^{-}\right)}, \\
& \left(0^{+}, 1^{+}\right) \rightarrow\left(0^{-}, 1^{-}\right): \quad \mathrm{S} \text { - wave transition, } \\
& A=\alpha_{\left(0^{+}, 1^{+}\right) \rightarrow\left(0^{-}, 1^{-}\right)} \gamma_{\mu} \partial_{\pi}^{\mu}, \\
& \left(1^{+}, 2^{+}\right) \rightarrow\left(0^{-}, 1^{-}\right): \quad \mathrm{D} \text { - wave transition, } \\
& A^{\nu}=\frac{i}{\Lambda_{\chi}} \alpha_{\left(1^{+}, 2^{+}\right) \rightarrow\left(0^{-}, 1^{-}\right)} \gamma_{\mu} \partial_{\pi}^{\mu} \partial_{\pi}^{\nu}, \\
& \left(1^{-}, 2^{-}\right) \rightarrow\left(0^{-}, 1^{-}\right): \quad \mathrm{P} \text { - wave transition, } \\
& A^{\nu}=\alpha_{\left(1^{-}, 2^{-}\right) \rightarrow\left(0^{-}, 1^{-}\right)} \partial_{\pi}^{\nu}, \\
& \left(1^{-}, 2^{-}\right) \rightarrow\left(1^{+}, 2^{+}\right): \quad \mathrm{S} \text { - wave transition, } \\
& A_{S}^{\mu \nu}=\alpha_{\left(1^{-}, 2^{-}\right) \rightarrow\left(1^{+}, 2^{+}\right)}^{(S)} g^{\mu \nu} v \cdot \partial_{\pi}, \\
& \mathrm{D} \text { - wave transition, } \\
& A_{D}^{\mu \nu}=\frac{i}{\Lambda_{\chi}} \alpha_{\left(1^{-}, 2^{-}\right) \rightarrow\left(1^{+}, 2^{+}\right)}^{(D)} \mathcal{P}_{\mu \nu \rho \sigma} \partial_{\pi}^{\rho} \partial_{\pi}^{\sigma},
\end{aligned}
$$

where $\mathcal{P}_{\mu \nu \rho \sigma} \equiv\left(g_{\mu \rho}-v_{\mu} v_{\rho}\right)\left(g_{\nu \sigma}-v_{\nu} v_{\sigma}\right)-\frac{1}{3}\left(g_{\mu \nu}-v_{\mu} v_{\nu}\right)\left(g_{\rho \sigma}-v_{\rho} v_{\sigma}\right)$, and $\partial_{\pi}$ indicates the partial derivative has to act on the pion field. The dimensionless effective couplings $\alpha$ will be extracted from our calculation in the chiral quark model. The scale $\Lambda_{\chi}$ will be taken to be $1 \mathrm{GeV}$. Both approaches necessarily reproduce the results of Eqn. (3). Only the effective coupling constants are not known. In this article, we use the chiral quark model, along with relativistic wave functions for the heavy mesons, to arrive at estimates of these couplings. We will also provide general results for the different partial wave decay widths. In what follows we work in the rest frame of the parent hadron. All expressions refer to that frame.

\section{THE CHIRAL QUARK MODEL}

In the calculation of the decay amplitudes we use the chiral quark model as proposed by Georgi and Manohar [5]. In this model the constituent light quarks acquire their masses from the spontaneous chiral symmetry breaking plus a contribution from the current quark masses that explicitly break chiral symmetry. The latter can be neglected in the case of the 
$u$ and $d$ quarks, but in the case of the $s$ quark it ought to be included. This means that, while the axial vector coupling $g_{A}^{q}$ of the constituent quark can be taken to be the same for all three flavors, the effect of the $\mathrm{SU}(3)$ breaking by the quark masses must be included in the Dirac equation of our quark model. Thus, chiral symmetry dictates the coupling of the Goldstone modes (pions) to the constituent quarks. In terms of constituent quark fields, the lowest order Lagrangian in the pion momentum expansion can be expressed as

$$
\begin{aligned}
\mathcal{L}_{C h Q M} & =i \bar{q} \gamma^{\mu} \nabla_{\mu} q-\bar{q} \tilde{m}_{q} q-\bar{q} V(x) q+g_{A}^{q} \bar{q} \gamma^{\mu} \gamma_{5} \omega_{\mu} q \\
\nabla_{\mu} & =\partial^{\mu}+i \Gamma^{\mu}, \quad \Gamma^{\mu}=\frac{i}{8 F_{\pi}}\left[\Pi, \partial^{\mu} \Pi\right]+\ldots \\
\omega^{\mu} & =\frac{1}{2 F_{\pi}} \partial^{\mu} \Pi+\ldots
\end{aligned}
$$

Here $q$ represents the triplet of light quarks $(u, d, s), \Gamma^{\mu}=\frac{i}{8 F_{\pi}}\left[\Pi, \partial^{\mu} \Pi\right]+\ldots$, and $g_{A}^{q}$ is the axial vector coupling of the constituent quark. The value of $g_{A}^{q}$ is not well known. Arguments in the large $\mathrm{N}_{c}$ limit show that $g_{A}^{q}=1+\mathcal{O}\left(1 / \mathrm{N}_{\mathrm{c}}\right) \sqrt{10}$, while an estimate of the nucleon axial coupling $g_{A}=1.255$ obtained with the chiral quark model in a non-relativistic approximation gives $g_{A}^{q} \simeq 0.75$ [5]. Since relativistic effects must increase the ratio $g_{A}^{q} / g_{A}$, we will consider $g_{A}^{q}$ between the two mentioned values. We work in the limit where the heavy quark is regarded as infinitely heavy, and $V(x)$ represents the interaction of the light valence quark with the heavy quark. Here we use the vector Coulomb plus linear scalar potentials with standard strengths for each piece.

We write the wave function of the light valence quark as

$$
\begin{aligned}
\psi_{j \ell m} & =\left(\begin{array}{c}
i F(r) \Omega_{j \ell m} \\
G(r) \Omega_{j \tilde{\ell} m}
\end{array}\right), \\
\tilde{\ell} & =2 j-\ell
\end{aligned}
$$

where the radial wave functions are real, and the spinor harmonics are given by

$$
\begin{aligned}
& \Omega_{j=\ell+\frac{1}{2} \ell m}=\left(\begin{array}{c}
\sqrt{\frac{j+m}{2 j}} Y_{\ell m-\frac{1}{2}} \\
\sqrt{\frac{j-m}{2 j}} Y_{\ell m+\frac{1}{2}}
\end{array}\right), \\
& \Omega_{j=\ell-\frac{1}{2} \ell m}=\left(\begin{array}{c}
\sqrt{\frac{j+1-m}{2(j+1)}} Y_{\ell m-\frac{1}{2}} \\
-\sqrt{\frac{j+m+1}{2(j+1)}} Y_{\ell m+\frac{1}{2}}
\end{array}\right) .
\end{aligned}
$$

Our conventions here correspond to those of Bjorken and Drell [11].

The pion emission amplitudes require the calculation of the matrix elements of the time and spatial components of the axial-vector current operator to which the pion couples according to Eqn. (\$). If $\vec{p}_{\pi}$ is the momentum of the pion, the matrix elements of the time component of the axial current we need to consider are

$$
\left\langle j^{\prime} \ell^{\prime} m^{\prime}\left|\gamma_{0} \gamma_{5} \exp \left(-i \vec{p}_{\pi} \cdot \vec{r}\right)\right| j \ell m\right\rangle
$$




$$
\begin{aligned}
& =\frac{4 \pi}{\sqrt{2 j^{\prime}+1}}(-1)^{\ell-\ell^{\prime}+j-j^{\prime}} \sum_{\ell_{\pi}, m_{\pi}}(-i)^{\ell_{\pi}} Y_{\ell_{\pi} m_{\pi}}^{*}\left(\hat{p}_{\pi}\right)\left\langle j m, \ell_{\pi} m_{\pi} \mid j^{\prime} m^{\prime}\right\rangle \\
& \times \int d r r^{2} j_{\ell_{\pi}}\left(p_{\pi} r\right)\left\{F^{\prime}(r) G(r)\left\langle j^{\prime} \ell^{\prime}\left\|A_{0}^{\ell_{\pi}}\right\| j \tilde{\ell}\right\rangle\right. \\
& \left.-G^{\prime}(r) F(r)\left\langle j^{\prime} \tilde{\ell}^{\prime}\left\|A_{0}^{\ell_{\pi}}\right\| j \ell\right\rangle\right\},
\end{aligned}
$$

where

$$
\begin{aligned}
\left\langle j^{\prime} \ell^{\prime}\left\|A_{0}^{\ell_{\pi}}\right\| j \ell\right\rangle & =\frac{(-1)^{j+\ell_{\pi}+\frac{1}{2}}}{\sqrt{4 \pi}}\left(\begin{array}{ccc}
\ell_{\pi} & \ell & \ell^{\prime} \\
0 & 0 & 0
\end{array}\right) \\
& \times \sqrt{(2 \ell+1)\left(2 \ell^{\prime}+1\right)\left(2 \ell_{\pi}+1\right)(2 j+1)\left(2 j^{\prime}+1\right)}\left\{\begin{array}{ccc}
\ell_{\pi} & \ell & \ell^{\prime} \\
\frac{1}{2} & j^{\prime} & j
\end{array}\right\} .
\end{aligned}
$$

Similarly, the matrix elements of the spatial components of the axial current are

$$
\begin{aligned}
& \left\langle j^{\prime} \ell^{\prime} m^{\prime}\left|\gamma_{i} \gamma_{5} \exp \left(-i \vec{p}_{\pi} \cdot \vec{r}\right)\right| j \ell m\right\rangle \\
= & 4 \pi(-1)^{\ell-\ell^{\prime}+j-j^{\prime}} \sum_{\ell_{\pi} m_{\pi}}(-i)^{\ell_{\pi}} Y_{\ell_{\pi} m_{\pi}}^{*}\left(\hat{p}_{\pi}\right) \\
\times & \sum_{\ell^{*} m^{*}}\left\langle 1 i, \ell_{\pi} m_{\pi} \mid \ell^{*} m^{*}\right\rangle\left\langle j-m, j^{\prime} m^{\prime} \mid \ell^{*} m^{*}\right\rangle \\
\times & \int d r r^{2} j_{\ell_{\pi}}\left(p_{\pi} r\right)\left\{F^{\prime}(r) F(r)\left\langle j^{\prime} \ell^{\prime}\left\|A^{\ell_{\pi}, \ell^{*}}\right\| j \ell\right\rangle\right. \\
- & \left.G^{\prime}(r) G(r)\left\langle j^{\prime} \tilde{\ell}^{\prime}\left\|A^{\ell_{\pi}, \ell^{*}}\right\| j \tilde{\ell}\right\rangle\right\},
\end{aligned}
$$

where

$$
\begin{aligned}
\left\langle j^{\prime} \ell^{\prime}\left\|A^{\ell_{\pi}, \ell^{*}}\right\| j \ell\right\rangle & =\sqrt{\frac{3}{2 \pi}} \sqrt{(2 \ell+1)\left(2 \ell^{\prime}+1\right)\left(2 \ell_{\pi}+1\right)(2 j+1)\left(2 j^{\prime}+1\right)} \\
& \times\left(\begin{array}{ccc}
\ell & \ell_{\pi} & \ell^{\prime} \\
0 & 0 & 0
\end{array}\right)\left\{\begin{array}{ccc}
\frac{1}{2} & \ell & j \\
\frac{1}{2} & \ell^{\prime} & j^{\prime} \\
1 & \ell_{\pi} & \ell^{*}
\end{array}\right\} .
\end{aligned}
$$

These expressions reflect the known fact that the time component of the axial current has matrix elements that are suppressed in the non-relativistic limit $\left(G(r), G^{\prime}(r) \rightarrow 0\right)$, while the matrix elements of the spatial components are not.

The general expression for the amplitudes is

$$
\left\langle J^{\prime} J_{3}^{\prime}, j^{\prime} \ell^{\prime} ; \pi^{a}, \vec{p}_{\pi} \mid J J_{3}, j \ell\right\rangle=\frac{i}{2 F_{\pi}} \xi^{a} g_{A}^{q} p_{\pi}^{\mu}\left\langle J^{\prime} J_{3}^{\prime}, j^{\prime} \ell^{\prime}\left|A_{\mu}^{a}\left(\vec{p}_{\pi}\right)\right| J J_{3}, j \ell\right\rangle,
$$

where $\xi^{a}$ is equal to $\sqrt{2}$ if $\pi^{a}=\pi^{ \pm}$and equal to 1 if $\pi^{a}=\pi^{0}$.

After performing the coupling of the angular momentum $j$ of the light quark to the heavy quark spin, one can express the above amplitude in a pion partial wave decomposition

$$
\begin{aligned}
\left\langle J^{\prime} J_{3}^{\prime}, j^{\prime} \ell^{\prime} ; \pi^{a}, \vec{p}_{\pi} \mid J J_{3}, j \ell\right\rangle & =\frac{i}{2 F_{\pi}} \xi^{a} g_{A}^{q} \sum_{\ell_{\pi} m_{\pi}}(-i)^{\ell_{\pi}} Y_{\ell_{\pi} m_{\pi}}^{*}\left(\hat{p_{\pi}}\right)\left\langle\ell_{\pi} m_{\pi}, J J_{3} \mid J^{\prime} J_{3}^{\prime}\right\rangle \\
& \times \mathcal{A}_{\ell_{\pi}}\left(p_{\pi} ; J, j \ell ; J^{\prime}, j^{\prime} \ell^{\prime}\right) .
\end{aligned}
$$


Here the amplitudes $\mathcal{A}_{\ell_{\pi}}\left(p_{\pi} ; J, j \ell ; J^{\prime}, j^{\prime} \ell^{\prime}\right)$ are obtained from (2.4) and (2.6) by performing the mentioned coupling of the heavy quark spin. These amplitudes are, explicitly

$$
\begin{aligned}
\mathcal{A}_{\ell_{\pi}} & =\mathcal{A}_{\ell_{\pi}}^{0}+\mathcal{A}_{\ell_{\pi}}^{1}, \\
\mathcal{A}_{\ell_{\pi}}^{0}\left(p_{\pi} ; J, j \ell ; J^{\prime}, j^{\prime} \ell^{\prime}\right) & =4 \pi i(-1)^{\ell-\ell^{\prime}+j-j^{\prime}} E_{\pi}(-1)^{J^{\prime}+j^{\prime}+\frac{1}{2}} \sqrt{2 J+1}\left\{\begin{array}{ccc}
\ell_{\pi} & j & j^{\prime} \\
\frac{1}{2} & J^{\prime} & J
\end{array}\right\} \\
& \times \int d r r^{2} j_{\ell_{\pi}}\left(p_{\pi} r\right)\left\{F^{\prime}(r) G(r)\left\langle j^{\prime} \ell^{\prime}\left\|A_{0}^{\ell_{\pi}}\right\| j \tilde{\ell}\right\rangle\right. \\
& \left.-G^{\prime}(r) F(r)\left\langle j^{\prime} \tilde{\ell}^{\prime}\left\|A_{0}^{\ell_{\pi}}\right\| j \ell\right\rangle\right\}, \\
\mathcal{A}_{\ell_{\pi}}^{1}\left(p_{\pi} ; J, j \ell ; J^{\prime}, j^{\prime} \ell^{\prime}\right) & =4 \pi(-1)^{\ell-\ell^{\prime}+j-j^{\prime}} p_{\pi} \sqrt{2 J+1}\left\{\begin{array}{ccc}
\ell_{\pi} & j & j^{\prime} \\
\frac{1}{2} & J^{\prime} & J
\end{array}\right\} \\
& \times \sum_{\ell^{*}}(-i)^{\ell^{*}-\ell_{\pi}}(-1)^{\ell_{\pi}+j-\frac{1}{2}+J^{\prime}-\ell^{*}} \sqrt{\left(2 \ell^{*}+1\right)\left(2 \ell_{\pi}+1\right)}\left(\begin{array}{ccc}
1 & \ell^{*} & \ell_{\pi} \\
0 & 0 & 0
\end{array}\right) \\
& \times(-1)^{j+j^{\prime}+\ell} \int d r r^{2} j_{\ell^{*}}\left(p_{\pi} r\right)\left\{F^{\prime}(r) F(r)\left\langle j^{\prime} \ell^{\prime}\left\|A^{\ell^{*}, \ell_{\pi}}\right\| j \ell\right\rangle\right. \\
& \left.-G^{\prime}(r) G(r)\left\langle j^{\prime} \tilde{\ell}^{\prime}\left\|A^{\ell^{*}, \ell_{\pi}}\right\| j \tilde{\ell}\right\rangle\right\} .
\end{aligned}
$$

These expressions are valid for emission of a light pseudoscalar from any heavy parent, in the chiral quark model. Note that both $\mathcal{A}_{\ell_{\pi}}^{0}$ and $\mathcal{A}_{\ell_{\pi}}^{1}$ explicitly contain the factors expected from heavy quark spin symmetry and spin counting arguments. This in turn serves as a check that our results satisfy the constraints implied by heavy quark spin symmetry. Also note that in this equation the reduced matrix element is evaluated with $\ell^{*}$ and $\ell_{\pi}$ interchanged with respect to the expression given in Eqn. (14).

With these expressions, the partial wave decay widths are obtained as

$$
\Gamma_{\ell_{\pi}}^{a}\left(J j \ell ; J^{\prime} j^{\prime} \ell^{\prime}\right)=\frac{2 J^{\prime}+1}{2 J+1} \frac{\xi^{a 2} g_{A}^{q 2} p_{\pi}}{32 \pi^{2} F_{\pi}^{2}} \frac{M_{J^{\prime}}}{M_{J}}\left|\mathcal{A}_{\ell_{\pi}}\left(p_{\pi} ; J, j \ell ; J^{\prime}, j^{\prime} \ell^{\prime}\right)\right|^{2} .
$$

Here the factor $\frac{M_{J^{\prime}}}{M_{J}}$ arises from the normalization of the heavy meson states.

Along similar lines one could consider the emission of a $\mathrm{K}$ meson in the decay of a strange excited heavy meson to a non-strange heavy meson. The flavor factor $\xi^{a}$ is in this case the same as for the emission of a charged pion, i.e., equal to $\sqrt{2}$. Also one could look into $\eta$ meson emission. In this case the factor $\xi^{a}$ is equal to $1 / \sqrt{3}$ for non-strange heavy mesons and equal to $-2 / \sqrt{3}$ for strange heavy mesons.

The results obtained above for $\mathcal{A}_{\ell_{\pi}}$ can be checked by performing the calculation in a different way. Indeed, the term linear in $\partial_{\mu} \Pi$ in $g_{A}^{q} \bar{q} \gamma^{\mu} \gamma_{5} \omega_{\mu} q$ in Eqn. (8) can be rewritten, after integration by parts and use of the Dirac equation in the potential $V(x)$, as

$$
i \frac{g_{A}^{q}}{2 F_{\pi}} \bar{q}\left\{\gamma_{5}, \tilde{m}_{q}+V(x)\right\} q .
$$

When one uses the vector Coulomb plus scalar linear potential, the result for $\mathcal{A}_{\ell_{\pi}}$ becomes

$$
\begin{aligned}
\mathcal{A}_{\ell_{\pi}}^{1}\left(p_{\pi} ; J, j \ell ; J^{\prime}, j^{\prime} \ell^{\prime}\right) & =4 \pi i(-1)^{\left(J^{\prime}+1 / 2+\ell-\ell^{\prime}+j\right)} \sqrt{2 J+1}\left\{\begin{array}{ccc}
\ell_{\pi} & j & j^{\prime} \\
\frac{1}{2} & J^{\prime} & J
\end{array}\right\} \\
& \times \int d r r^{2} j_{\ell_{\pi}}\left(p_{\pi} r\right) 2\left(\tilde{m}_{q}+K r\right)\left\{F^{\prime}(r) G(r)\left\langle j^{\prime} \ell^{\prime}\left\|A_{0}^{\ell_{\pi}}\right\| j \tilde{\ell}\right\rangle\right. \\
& \left.+G^{\prime}(r) F(r)\left\langle j^{\prime} \tilde{\ell}^{\prime}\left\|A_{0}^{\ell_{\pi}}\right\| j \ell\right\rangle\right\} .
\end{aligned}
$$


Clearly, since the two expressions obtained for $\mathcal{A}_{\ell_{\pi}}$ only coincide when the Dirac equation for the bound light quark is satisfied, we can use them as a check that the numerical solutions for the wave functions are sufficiently precise.

The effective couplings in Eqn. (7) are also readily obtained by matching the amplitudes in the superfield formalism with the amplitudes obtained in the chiral quark model. For convenience the matching is done with the latter multiplied by $i^{\ell_{\pi}}$, and we obtain:

$$
\begin{aligned}
& \left(0^{-}, 1^{-}\right) \rightarrow\left(0^{-}, 1^{-}\right): \quad \mathrm{P} \text { - wave transition } \\
& \alpha_{\left(0^{-}, 1^{-}\right) \rightarrow\left(0^{-}, 1^{-}\right)}=-g_{A}^{q}\left\{\int d r r^{2}\left[j_{0}\left(p_{\pi} r\right)\left(F^{\prime}(r) F(r)-\frac{1}{3} G^{\prime}(r) G(r)\right)-\frac{4}{3} j_{2}\left(p_{\pi} r\right) G^{\prime}(r) G(r)\right]\right. \\
& \left.+\frac{E_{\pi}}{p_{\pi}} \int d r r^{2} j_{1}\left(p_{\pi} r\right)\left(F^{\prime}(r) G(r)-G^{\prime}(r) F(r)\right)\right\} ; \\
& \left(0^{+}, 1^{+}\right) \rightarrow\left(0^{-}, 1^{-}\right): \quad \mathrm{S}-\text { wave transition } \\
& \alpha_{\left(0^{+}, 1^{+}\right) \rightarrow\left(0^{-}, 1^{-}\right)}=-g_{A}^{q}\left\{\int d r r^{2} j_{0}\left(p_{\pi} r\right)\left(F^{\prime}(r) G(r)-G^{\prime}(r) F(r)\right)\right. \\
& \left.-\frac{p_{\pi}}{E_{\pi}} \int d r r^{2} j_{1}\left(p_{\pi} r\right)\left(F^{\prime}(r) F(r)+G^{\prime}(r) G(r)\right)\right\} \text {; } \\
& \left(1^{+}, 2^{+}\right) \rightarrow\left(0^{-}, 1^{-}\right): \quad \mathrm{D}-\text { wave transition } \\
& \alpha_{\left(1^{+}, 2^{+}\right) \rightarrow\left(0^{-}, 1^{-}\right)}=\Lambda_{\chi} \sqrt{3} g_{A}^{q}\left\{-\frac{E_{\pi}}{p_{\pi}^{2}} \int d r r^{2} j_{2}\left(p_{\pi} r\right)\left(F^{\prime}(r) G(r)-G^{\prime}(r) F(r)\right)\right. \\
& \left.-\frac{1}{p_{\pi}} \int r^{2} d r\left[j_{1}\left(p_{\pi} r\right)\left(F^{\prime}(r) F(r)-\frac{1}{5} G^{\prime}(r) G(r)\right)+\frac{6}{5} j_{3}\left(p_{\pi} r\right) G^{\prime}(r) G(r)\right]\right\} \\
& \left(1^{-}, 2^{-}\right) \rightarrow\left(0^{-}, 1^{-}\right): \quad \mathrm{P} \text { - wave transition } \\
& \alpha_{\left(1^{-}, 2^{-}\right) \rightarrow\left(0^{-}, 1^{-}\right)}=\sqrt{3} g_{A}^{q}\left\{\frac{E_{\pi}}{p_{\pi}} \int d r r^{2} j_{1}\left(p_{\pi} r\right)\left(F^{\prime}(r) G(r)-G^{\prime}(r) F(r)\right)\right. \\
& \left.+\int r^{2} d r\left[\frac{2}{3} j_{0}\left(p_{\pi} r\right) G^{\prime}(r) G(r)-j_{2}\left(p_{\pi} r\right)\left(F^{\prime}(r) F(r)+\frac{1}{3} G^{\prime}(r) G(r)\right)\right]\right\} ; \\
& \left(1^{-}, 2^{-}\right) \rightarrow\left(1^{+}, 2^{+}\right): \\
& \mathrm{S} \text { - wave transition } \\
& \left.\alpha_{\left(1^{-}, 2^{-}\right) \rightarrow\left(1^{+}, 2^{+}\right)}\right|_{S}=-g_{A}^{q}\left\{\int d r r^{2} j_{0}\left(p_{\pi} r\right)\left(F^{\prime}(r) G(r)-G^{\prime}(r) F(r)\right)\right. \\
& \left.-\frac{p_{\pi}}{E_{\pi}} \int d r r^{2} j_{1}\left(p_{\pi} r\right)\left(F^{\prime}(r) F(r)+G^{\prime}(r) G(r)\right)\right\} \\
& \text { D - wave transition } \\
& \left.\alpha_{\left(1^{-}, 2^{-}\right) \rightarrow\left(1^{+}, 2^{+}\right)}\right|_{D}=-\Lambda_{\chi} 3 g_{A}^{q}\left\{-\frac{E_{\pi}}{p_{\pi}^{2}} \int d r r^{2} j_{2}\left(p_{\pi} r\right)\left(F^{\prime}(r) G(r)-G^{\prime}(r) F(r)\right)\right. \\
& -\frac{2}{5 p_{\pi}} \int r^{2} d r\left[j_{1}\left(p_{\pi} r\right)\left(F^{\prime}(r) F(r)+G^{\prime}(r) G(r)\right)\right. \\
& \left.\left.+\frac{3}{5 p_{\pi}} j_{3}\left(p_{\pi} r\right)\left(F^{\prime}(r) F(r)+G^{\prime}(r) G(r)\right)\right]\right\} \text {. }
\end{aligned}
$$


Note that in the non-relativistic limit $\left(G(r), G^{\prime}(r) \rightarrow 0\right)$, the expression for $\alpha_{\left(0^{-}, 1^{-}\right) \rightarrow\left(0^{-}, 1^{-}\right)}$ gives the coupling of a pion to the ground state heavy mesons $g=g_{A}^{q}$; this is a factor two larger than the one used in [3], and is due to the different normalization of the heavy meson fields we used there.

In terms of the effective coupling constants, the decay widths for emission of a $\pi^{0}$ are

$$
\begin{aligned}
& \Gamma_{\left(0^{-}, 1^{-}\right) \rightarrow\left(0^{-}, 1^{-}\right)}=\frac{\left|\alpha_{\left(0^{-}, 1^{-}\right) \rightarrow\left(0^{-}, 1^{-}\right)}\right|^{2}}{24 \pi F_{\pi}^{2}} \frac{M_{f}}{M_{i}}\left|\vec{p}_{\pi}\right|^{3}, \\
& \Gamma_{\left(0^{+}, 1^{+}\right) \rightarrow\left(0^{-}, 1^{-}\right)}=\frac{\left|\alpha_{\left(0^{+}, 1^{+}\right) \rightarrow\left(0^{-}, 1^{-}\right)}\right|^{2}}{8 \pi F_{\pi}^{2}} \frac{M_{f}}{M_{i}} E_{\pi}^{2}\left|\vec{p}_{\pi}\right|, \\
& \Gamma_{\left(1^{+}, 2^{+}\right) \rightarrow\left(0^{-}, 1^{-}\right)}=\frac{\left|\alpha_{\left(1^{+}, 2^{+}\right) \rightarrow\left(0^{-}, 1^{-}\right)}\right|^{2}}{24 \pi F_{\pi}^{2} \Lambda_{\chi}^{2}} \frac{M_{f}}{M_{i}}\left|\vec{p}_{\pi}\right|^{5}, \\
& \Gamma_{\left(1^{-}, 2^{-}\right) \rightarrow\left(0^{-}, 1^{-}\right)}=\frac{\left|\alpha_{\left(1^{-}, 2^{-}\right) \rightarrow\left(0^{-}, 1^{-}\right)}\right|^{2}}{36 \pi F_{\pi}^{2}} \frac{M_{f}}{M_{i}}\left|\vec{p}_{\pi}\right|^{3}, \\
& \Gamma_{\left(1^{-}, 2^{-}\right) \rightarrow\left(1^{+}, 2^{+}\right)}^{S-\text { wave }}=\frac{\left|\alpha_{\left(1^{-}, 2^{-}\right) \rightarrow\left(1^{+}, 2^{+}\right)}\right|^{2}}{8 \pi F_{\pi}^{2}} \frac{M_{f}}{M_{i}} E_{\pi}^{2}\left|\vec{p}_{\pi}\right|, \\
& \Gamma_{\left(1^{-}, 2^{-}\right) \rightarrow\left(1^{+}, 2^{+}\right)}^{D-\text { wave }}=\frac{\left|\alpha_{\left(1^{-}, 2^{-}\right) \rightarrow\left(1^{+}, 2^{+}\right)}\right|^{2}}{144 \pi F_{\pi}^{2} \Lambda_{\chi}^{2}} \frac{M_{f}}{M_{i}}\left|\vec{p}_{\pi}\right|^{5},
\end{aligned}
$$

where $M_{f}$ is the mass of the heavy daughter hadron, and $M_{i}$ is the mass of the decaying parent. Except for the transitions within the ground state multiplet for which isospin breaking plays a crucial role, for all other cases the total decay width into pions (ie, including neutral and charge pion modes) is three times the width given in Eqn. (22).

\section{RESULTS AND DISCUSSION}

The wave functions that we use for this calculation are obtained from previous work [12]. In [12], the heavy meson spectrum is obtained by solving the one-body Dirac equation that arises from the Gross reduction of the Bethe-Salpeter equation. The potential used in that calculation consisted of a scalar confining and vector Coulomb potential, and the spectra obtained were in good agreement with experimental measurements. In addition, the wave functions and the Bethe-Salpeter formalism have been applied to a calculation of the Isgur-Wise function [13], and preliminary results indicate that the method compares well to others.

The wave functions in question have been obtained using two different methods. In the first, the two components of the Dirac equation are combined to produce a single second order differential equation, which is solved using standard numerical techniques. We refer to the wave functions obtained in this way as the 'exact' wave function. In the second method, the wave functions are expanded in a finite-sized basis of orthogonal functions, and a matrix diagonalization, as well as minimization in the energy eigenvalues are performed. The size of the basis is varied to examine the sensitivity of our model predictions to the size of the expansion basis. We have included the predictions for the heavy meson spectrum for the states relevant for this work, in table 1 . 
Our results for the coupling constants obtained in this model are shown in table $\mathbb{1} \mathbb{1}$. The integrals in Eqn. (21) depend on the momentum of the light daughter pseudoscalar, and are therefore 'form factors' rather than 'coupling constants'. The results in the table are obtained using $q^{2}=m_{\pi}^{2}$ for all but the intra-multiplet transitions of the $\left(0^{-}, 1^{-}\right)$multiplet, since most of these transitions are kinematically forbidden. For these, we use $q^{2}=10^{-4}$ $\mathrm{GeV}^{2}$. Note that we show signs on the coupling constants in this table, but we remind the reader that the signs we obtain depend on the sign conventions used for the wave functions, and are therefore not physically meaningful. What is meaningful is the relative sign between the S- and D-wave coupling constants in the fourth and fifth rows of the table.

We note that for most of the decays we consider, the coupling constants show little sensitivity to the mass of the heavy quark, with the largest effect being of the order of $10 \%$ as we go from $c$-flavored to $b$-flavored mesons. The exception here are the decays of the radially excited $\left(0^{-}, 1^{-}\right)$states. In addition, again with the exception of the radial excitations of the ground state, $\mathrm{SU}(3)$ breaking effects are quite small. The reader is reminded that there are three possible sources of SU(3) breaking in our model. First, the strange quark mass used in obtaining the wave functions is different from that of the up and down quarks. Second, the (SU(3) broken) physical masses of the hadrons are used in calculating the coupling constants, and third, in the case of the decay widths, the ( $\mathrm{SU}(3)$ broken) physical masses are used in the calculation of phase space. In the case of the coupling constants, only the first two sources come into play, but we see from our results that the net effect is very small. It is nevertheless interesting to note that the heavy quark symmetry appears to be a better symmetry.

Comparison of these numbers with those that we reported earlier clearly show the importance of relativistic effects. This is especially evident for the transitions within the $\left(0^{-}, 1^{-}\right)$ multiplet. In the non-relativistic limit, this coupling constant, denoted $g$, has the value of 1 , in units of $g_{A}^{q}$. In the present calculation, modulo a sign that arises from the definition, this coupling constant ranges from 0.74 to 0.79 , in the same units. Relativistic effects also become evident when we examine the decay widths obtained in the model.

Our results for the decay widths of excited mesons, obtained using the chiral quark model, are shown in table III. In this table, all numbers are in $\mathrm{MeV} /\left(g_{A}^{q 2}\right)$. The numbers in the columns labeled $D$ and $B$ arise from pion emission, while those in the columns labeled $D_{s}$ and $B_{s}$ arise from kaon emission. Blank entries in the table correspond to decays that are kinematically forbidden. For observed states, we use the measured masses in calculating phase space. For states that have not yet been observed, we use the model predictions for the masses. Our estimates for the theoretical errors that we show are obtained by varying the masses of the parent hadrons by $\pm 20 \mathrm{MeV}$, and calculating the decay rates at the new masses.

The results that we report are obtained using the approximate wave functions described above. For comparison, we have used 5, 10 and 15 basis functions in the wave function expansion, and found that the results for the widths do not vary significantly. In fact, we find a change of about $2 \mathrm{MeV} /\left(g_{A}^{q 2}\right)(\simeq 2 \%)$ in the largest widths we report, those for $0^{+} \rightarrow 0^{-}$. All variations we observe are of the order of or less than $3 \%$.

For the intra-multiplet transition of the $\left(0^{-}, 1^{-}\right)$multiplet our results are encouraging, but suggest that $g_{A}^{q}$ should be less than unity. This is in keeping with other articles that suggest a value for this constant as small as 0.7 . Using the latest total width reported for the 
$D^{*+}\left(\Gamma_{D^{* \pm}}<0.131 \mathrm{MeV}\right)$, we find $\left|g_{A}^{q}\right|<0.95$. Using this upper limit, and the published ratio of the strong to electromagnetic widths of the $D^{* 0}$, we estimate that $\Gamma_{D^{* 0}}<0.094$ $\mathrm{MeV}$. The current experimental limit is $\Gamma_{D^{* 0}}<2.1 \mathrm{MeV}$.

The decays of the $\left(1^{+}, 2^{+}\right)$doublet would appear to require a value for $\left|g_{A}^{q}\right|$ near the lower limit of 0.7 , in order for our results to be consistent with the latest widths reported in the particle listings $\left(\Gamma_{D_{1}^{0}}=18.9_{-3.5}^{+4.6} \mathrm{MeV}, \Gamma_{D_{1}^{ \pm}}=28 \pm 8 \mathrm{MeV}, \Gamma_{D_{2}^{0}}=23 \pm 5 \mathrm{MeV}\right.$, $\Gamma_{D_{2}^{ \pm}}=25_{-9}^{+8} \mathrm{MeV}$ ), [14]. We remind the reader that, except for the decay within the ground-state multiplet, we do not consider $\mathrm{SU}(2)$ breaking effects in our calculation.

There are large uncertainties on the measured widths, and the total widths of the $D_{1}$ suggest that $\left|g_{A}^{q}\right|>1$. It is known, however, that the decays of the $D_{1}$ are not well described in a heavy quark symmetry (HQS) framework, unless $1 / m_{Q}$ corrections are taken into account. In particular, an S-wave contribution to the decay of this state can be expected [15]. On the other hand, the decays of the $D_{2}$ have been well described in the HQS framework, without the need for $1 / m_{Q}$ corrections. For instance, we obtain

$$
\frac{\Gamma_{D_{2} \rightarrow D \pi}}{\Gamma_{D_{2} \rightarrow D^{*} \pi}}=1.93,
$$

while the measured values are $2.3 \pm 0.6$ for the neutral $D_{2}$, and $1.9 \pm 1.1 \pm 0.3$ for the charged. If we crudely average the measure widths of the $D_{2}$ for mixed charged states to $24 \mathrm{MeV}$, our model would require $\left|g_{A}^{q}\right|=0.74$. This would then lead to $\Gamma_{D^{* \pm}}=0.082 \mathrm{MeV}$ and $\Gamma_{D^{* 0}}=$ $0.059 \mathrm{MeV}$.

As expected from previous work, our S-wave decay widths $\left(0^{+} \rightarrow 0^{-}, 1^{+} \rightarrow 1^{-}, 1^{-} \rightarrow 1^{+}\right.$ and $2^{-} \rightarrow 2^{+}$) are relatively large, but much smaller than the values obtained in nonrelativistic calculations, indicating that relativistic effects are significant for these decays. In fact, for all of our results, the decay widths that we obtain are significantly smaller than the numbers we obtained using a non-relativistic version of the chiral quark model [3]. For the decay $0^{+} \rightarrow 0^{-}$, the result we obtain in the present model is about one-eighth of the width reported in [3]. The reduction of S-wave amplitudes by relativistic effects has also been seen in other model calculations [16]. For the radially excited pseudoscalar-vector doublet, the decay widths we obtain are about fifteen percent of those presented in [3]: these states may be much narrower than we originally predicted. It will be interesting to see what effects these differences in widths will have on the spectrum expected in $B_{\ell 4}$ decays.

For the strange mesons, we find that phase space suppresses or forbids many of the possible decays. This suggests that many of the $D_{s}$ and $B_{s}$ excited mesons will be quite narrow, as their primary mode of decay will be radiative, as well as through isospin-violating, OZI-rule-violating pion emission. This 'double-suppression' means that these decay widths should be quite small, comparable to (or even smaller than) the radiative decay widths of these states.

The results of this work derive from the strict use of the chiral quark model, and the experimental determinations of the widths would provide a valuable test of this model. In particular, we have seen that S-wave decay widths are particularly sensitive to the model chosen. In summary, it would appear that the decays of excited heavy mesons provide the best testing ground for the chiral quark model. 


\section{ACKNOWLEDGEMENT}

WR gratefully acknowledges the hospitality and support of Université Joseph Fourier, Grenoble, France, and of Institut des Sciences Nucléaires, Grenoble, France, where part of this work was done, and both authors thank the Department of Physics of Florida State University, Tallahassee, and Dr. Simon Capstick in particular, for their kind hospitality while this work was being completed. This work was supported by the National Science

Foundation through grant \# HRD-9633750 (JLG) and \# PHY 9457892 (WR), and by the Department of Energy through contracts DE-AC05-84ER40150 (JLG and WR) and DEFG05-94ER40832 (WR). 


\section{TABLES}

\begin{tabular}{lllll}
\hline \hline \multirow{2}{*}{ State } & \multicolumn{5}{c}{ Mass $(\mathrm{GeV})$} \\
\cline { 2 - 5 } & $D$ & $B$ & $D_{s}$ & $B_{s}$ \\
\hline $0^{-}$ & $1.864(1.85)$ & $5.279(5.28)$ & $1.969(1.94)$ & $5.369(5.37)$ \\
$1^{-}$ & $2.007(2.02)$ & $5.325(5.33)$ & $2.114(2.13)$ & $5.416(5.43)$ \\
\hline $0^{+}$ & 2.27 & 5.65 & 2.38 & 5.75 \\
$1^{+}$ & 2.40 & 5.69 & 2.51 & 5.79 \\
\hline $1^{+}$ & $2.427(2.41)$ & 5.69 & $2.535(2.52)$ & 5.79 \\
$2^{+}$ & $2.459(2.46)$ & 5.71 & $2.573(2.58)$ & 5.82 \\
\hline $1^{-}$ & 2.71 & 5.97 & 2.82 & 6.07 \\
$2^{-}$ & 2.74 & 5.96 & 2.86 & 6.07 \\
\hline $0^{-\prime}$ & 2.50 & 5.83 & 2.61 & 5.93 \\
$1^{-\prime}$ & 2.62 & 5.87 & 2.73 & 5.97 \\
\hline
\end{tabular}

TABLE I. Masses of states obtained in the relativistic model. For observed states, the model masses are shown in parentheses.

\begin{tabular}{lrrrr}
\hline \hline Decay & \multicolumn{4}{c}{ (in units of $g_{A}^{q}$ ) } \\
\cline { 2 - 5 } & $D$ & $B$ & $D_{s}$ & $B_{s}$ \\
\hline$\left(0^{+}, 1^{+}\right) \rightarrow\left(0^{-}, 1^{-}\right)$ & 0.48 & 0.48 & 0.46 & 0.46 \\
$\left(1^{+}, 2^{+}\right) \rightarrow\left(0^{-}, 1^{-}\right)$ & -0.86 & -0.88 & -0.84 & -0.86 \\
$\left(1^{-}, 2^{-}\right) \rightarrow\left(0^{-}, 1^{-}\right)$ & 0.138 & 0.138 & 0.128 & 0.128 \\
{$\left[\left(1^{-}, 2^{-}\right) \rightarrow\left(1^{+}, 2^{+}\right)\right]_{S}$} & -0.50 & -0.50 & -0.48 & -0.48 \\
{$\left[\left(1^{-}, 2^{-}\right) \rightarrow\left(1^{+}, 2^{+}\right)\right]_{D}$} & -1.08 & -1.08 & -1.04 & -1.04 \\
$\left(0^{-}, 1^{-}\right)^{\prime} \rightarrow\left(0^{-}, 1^{-}\right)$ & 0.112 & 0.142 & 0.100 & 0.128 \\
$\left(0^{-}, 1^{-}\right) \rightarrow\left(0^{-}, 1^{-}\right)$ & -0.74 & -0.76 & -0.78 & -0.78 \\
\hline \hline
\end{tabular}

TABLE II. Coupling 'constants' obtained in the chiral quark model. In the columns labeled $B$ and $D$, pions are emitted in the decays, while in the columns labeled $D_{s}$ and $B_{s}$, the emitted mesons are kaons. 


\begin{tabular}{|c|c|c|c|c|c|}
\hline \multirow[t]{2}{*}{ Decay } & \multirow[t]{2}{*}{$\ell_{\pi}$} & \multicolumn{4}{|c|}{ ( width $\left(\mathrm{MeV} / g_{A}^{q 2}\right)$} \\
\hline & & $D$ & $B$ & $D_{s}$ & $B_{s}$ \\
\hline$\overline{0^{+} \rightarrow 0^{-}}$ & 0 & $125 \pm 10$ & $133 \pm 13$ & - & - \\
\hline $1^{+} \rightarrow 1^{-}$ & 0 & $121 \pm 10$ & $129 \pm 14$ & - & - \\
\hline$\overline{1^{+} \rightarrow 1^{-}}$ & 2 & $17.7 \pm 4.1$ & $12.5 \pm 3.9$ & - & - \\
\hline $2^{+} \rightarrow 0^{-}$ & 2 & $28.6 \pm 3.7$ & $11.4 \pm 2.7$ & $1.9 \pm 1.0$ & - \\
\hline $2^{+} \rightarrow 1^{-}$ & 2 & $14.8 \pm 3.0$ & $9.9 \pm 2.8$ & - & - \\
\hline$\overline{1^{-} \rightarrow 0^{-}}$ & 1 & $14.0 \pm 0.7$ & $13.4 \pm 1.2$ & $15.0 \pm 1.0$ & $10.5 \pm 1.8$ \\
\hline $1^{-} \rightarrow 1^{-}$ & 1 & $4.5 \pm 0.4$ & $5.3 \pm 0.6$ & $3.9 \pm 0.6$ & $3.7 \pm 0.7$ \\
\hline $2^{-} \rightarrow 1^{-}$ & 1 & $15.4 \pm 1.3$ & $15.1 \pm 1.8$ & $14.9 \pm 1.7$ & $10.9 \pm 2.2$ \\
\hline$\overline{1^{-} \rightarrow 1^{+}}$ & 0 & $52.8 \pm 6.8$ & $57.5 \pm 7.8$ & - & - \\
\hline $1^{-} \rightarrow 1^{+}$ & 2 & $0.57 \pm 0.21$ & $0.66 \pm 0.36$ & - & - \\
\hline $1^{-} \rightarrow 2^{+}$ & 2 & $0.26 \pm 0.16$ & $0.40 \pm 0.26$ & - & - \\
\hline $2^{-} \rightarrow 1^{+}$ & 2 & $0.63 \pm 0.27$ & $0.31 \pm 0.19$ & - & - \\
\hline $2^{-} \rightarrow 2^{+}$ & 0 & $52.3 \pm 6.7$ & $45.9 \pm 7.4$ & - & - \\
\hline $2^{-} \rightarrow 2^{+}$ & 2 & $0.77 \pm 0.40$ & $0.43 \pm 0.32$ & - & - \\
\hline$\overline{0^{-\prime} \rightarrow 1^{-}}$ & 1 & $26.1 \pm 1.8$ & $34.3 \pm 2.2$ & $0.13_{-0.13}^{+1.40}$ & $1.7_{-1.7}^{+2.8}$ \\
\hline $1^{-\prime} \rightarrow 0^{-}$ & 1 & $10.6 \pm 0.3$ & $13.5 \pm 0.3$ & $9.5 \pm 0.4$ & $5.5 \pm 1.3$ \\
\hline $1^{-\prime} \rightarrow 1^{-}$ & 1 & $22.4 \pm 0.5$ & $25.3 \pm 1.1$ & $9.5 \pm 1.8$ & $5.2 \pm 2.4$ \\
\hline$\overline{D^{*+}} \rightarrow D^{+} \pi^{0}$ & 1 & 0.045 & - & - & - \\
\hline$D^{*+} \rightarrow D^{0} \pi^{+}$ & 1 & 0.101 & - & - & - \\
\hline$D^{* 0} \rightarrow D^{0} \pi^{0}$ & 1 & 0.065 & - & - & - \\
\hline
\end{tabular}

TABLE III. Decay widths of heavy mesons obtained in the chiral quark model. In the columns labeled $B$ and $D$, pions are emitted in the decays, while in the columns labeled $D_{s}$ and $B_{s}$, the emitted mesons are kaons. All widths shown are in $\mathrm{MeV}$, and require a factor of $g_{A}^{q 2}$. Blank entries in the table correspond to decays that are kinematically forbidden. 


\section{REFERENCES}

[1] N. Isgur and M. Wise, Phys. Lett. B 232 (1989) 113, and, Phys. Lett. B 237 (1990) 527.

[2] J. D. Bjorken, I. Dunietz and J. Taron, Nucl.Phys. B 371 (1992) 111.

[3] J. L. Goity and W. Roberts, Phys. Rev. D 51 (1995) 3459.

[4] R. Akers et al., Z. Phys. bf C 67 (1995) 57.

D. Buskulic et al., Z. Phys. bf C 73 (1997) 601.

T.E. Browder et al., ICHEP '96 Proceedings. Edited by Z. Ajduk and A.K. Wroblewski. World Scientific (1997) 1139.

[5] H. Georgi and A. V. Manohar, Nucl. Phys. B 234 (1984) 189.

[6] A. F. Falk, Nucl.Phys. B 378 (1992) 79.

A. F. Falk and M. Luke, Phys. Lett. B 292 (1992) 119.

[7] G. Burdman and J. F. Donoghue, Phys. Lett. B 280 (1992) 287.

M. B. Wise, Phys. Rev. D 45 (1992) 2188.

T. M. Yan et al, Phys. Rev. D 46 (1992) 1148.

J. L. Goity, Phys. Rev. D 46 (1992) 3929.

[8] N. Isgur and M. Wise, Phys. Rev. Lett. 66 (1991) 1130.

[9] W. Roberts, Phys. Rev. D58 (1998).

[10] S. Weinberg, Phys. Rev. Lett. 67 (1991) 3473.

[11] J. D. Bjorken and S. D. Drell, Relativistic Quantum Mechanics, McGraw-Hill (1964).

[12] J. W. Van Orden, J. Zeng and W. Roberts, Phys. Rev. D52 (1995) 5229.

[13] J. W. Van Orden and W. Roberts, in preparation.

[14] C. Caso et al., European Physical Journal C (1998) 1.

[15] A. F. Falk and T. Mehen, Phys. Rev D53 (1996) 231; A. F. Falk, in Heidelberg 1996, Current Problems in Particle Theory: Non-Perturbative Particle Theory and Experimental Tests, Heidelberg, Germany, June 1996.

[16] S. Godfrey and R. Kokoski, Phys. Rev. D43 (1991) 1679. 